\title{
Prediction of clinical mastitis outcomes within and between environments using whole-genome markers
}

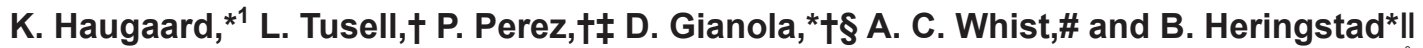 \\ *Department of Animal and Aquacultural Sciences, Norwegian University of Life Sciences, PO Box 5003, N-1432 Ås, Norway \\ †Department of Animal Sciences, University of Wisconsin-Madison, Madison 53706 \\ ¥Colegio de Postgraduados, Montecillo, Edo. de México, Mexico \\ $\S$ Departments of Dairy Science, and Biostatistics and Medical Informatics, University of Wisconsin-Madison, Madison 53706 \\ \#The Norwegian School of Veterinary Science, PO Box 8146 Dep, N-0033 Oslo, Norway \\ $\|$ Geno Breeding and Al Association, PO Box 5003, N-1432 Ås, Norway
}

\begin{abstract}
The objective of this study was to evaluate genomeenabled predictions of daughter yield deviations for clinical mastitis in Norwegian Red cows within and between environments according to mastitis pathogen status. Genome-based predictions of daughter yield deviations for clinical mastitis for 1,126 bulls within and between 5 environments were performed using Bayesian ridge regression. The environments were defined as herd-5-yr classes with the following prevalence of bacteriological milk samples found positive for contagious mastitis pathogens: $<50 \%$ (L50), $\geq 50 \%$ (H50), $\leq 25 \%$ (L75), $>25 \%$ and $<75 \%$ (M75), and $\geq 75 \%$ (H75). In addition, predictions based on all data across environment groups (the full data set, FD) were calculated to provide a benchmark for comparison. Predictive ability was evaluated using a 10 -fold cross validation. A bootstrap procedure was used to obtain $95 \%$ confidence intervals for the cross-validation distribution of predictive ability for each data set. Predictive ability ranged from 0.04 for L75 to 0.19 for FD. Similar predictions within and between environments showed no evidence of genotype by environment interaction. The $95 \%$ confidence interval for all 5 environmental data sets included zero and ranged from 0.02 to 0.35 for FD. The bootstrap distribution showed large variation within each data set and small variation between data sets. Although we found no evidence of genotype by environment interaction, rank correlations of the single nucleotide polymorphism effects between different environments ranged from 0.15 (L75 - H75) to 0.92 (M75 - FD), indicating that single nucleotide polymorphisms may have a differential contribution to predictive ability in environments with distinct pathogen loads.
\end{abstract}

Received September 6, 2012

Accepted February 26, 2013.

${ }^{1}$ Corresponding author: katrine.haugaard@umb.no
Key words: pathogen-specific mastitis, genomic prediction, genotype by environment interaction

\section{INTRODUCTION}

Clinical mastitis (CM) is the most common disease in dairy cattle and is of great concern in the dairy industry, mostly because of its adverse economic effects. Several different pathogens cause CM and the various pathogens affect the severity and duration of the disease differently. Pathogen prevalence varies among countries, regions, herds, and cattle breeds. The mastitis-causing pathogens can be divided into 2 groups, contagious and environmental, based on their distribution characteristics and interaction with the mammary gland. The contagious pathogens include Staphylococcus aureus, Streptococcus dysgalactiae, and Streptococcus agalactiae. These are present in the mammary gland (Calvinho et al., 1998) and transmitted between cows via milking equipment, operators, or by direct contact (Harmon, 1994). Environmental pathogens include Escherichia coli, Corynebacterium bovis, Streptococcus uberis, and CNS, and these have bedding, manure, and soil as their primary reservoirs (Harmon, 1994; Calvinho et al., 1998). An issue is whether these 2 types of pathogens produce genetically heterogeneous disease. Genetic correlations among CM and pathogenspecific CM of $<1$ have been estimated (Sørensen et al., 2009; Haugaard et al., 2012), suggesting that the phenotypic expression of CM caused by different pathogens may be controlled by different genes. Sørensen et al. (2008) reported that QTL associated with CM and SCC showed pathogen-specific effects, albeit with some uncertainty. Given that pathogen-specific genes exist, genotype by environment interaction $(\mathbf{G} \times \mathbf{E})$ for mastitis is an interesting issue. Using whole-genome markers is one possible approach to further investigate the subject.

If $\mathrm{G} \times \mathrm{E}$ exists, different genotypes may perform and rank differently in various environments. The question 
of what environment is optimum for selection is important and respective early studies were those of Falconer (1952, 1960) and Falconer and Latyszewski (1952). Using pedigree information has so far been the standard approach for investigating $\mathrm{G} \times \mathrm{E}$. With the advent of molecular markers, it is now possible to study $\mathrm{G} \times \mathrm{E}$ at the genomic level in humans (e.g., Neuman et al., 2002; Wang et al., 2002), livestock (e.g., Lillehammer et al., 2008; Long et al., 2008), and crops (e.g., Wei et al., 2010). Several methods have been used to investigate $\mathrm{G} \times \mathrm{E}$; for example, studies of specific genes (e.g., Neuman et al., 2002), molecular marker association studies (e.g., Wei et al., 2010), QTL studies (Lillehammer et al., 2008), or subsets of SNP selected based on information gain metrics (e.g., Long et al., 2008). Another approach is to use all available SNP in a statistical model and then compare predictive ability in different environments, as done by Burgueno et al. (2012) in plants.

The objective of this study was to evaluate genomeenabled predictions of daughter yield deviations (DYD) for CM in Norwegian Red cows within and between defined environments with herds classified into environments according to mastitis pathogen status. The aim was to investigate whether training the statistical model in one environment and testing it in another modified the predictive ability for DYD relative to that obtained when training and testing the model in the same environment. If this were the case, it would indicate that $\mathrm{G} \times \mathrm{E}$ exists for $\mathrm{CM}$, with the environment defined by a certain grouping of pathogens and the genotypes represented by half-sib families of Norwegian Red. Predictions based on all data across environmental groups were calculated as well to provide a benchmark for comparison.

\section{MATERIALS AND METHODS}

\section{Definition of Environment}

Because the average herd size in Norway is small (22 cows per herd), contemporary groups of herd5 -yr (H5Y) classes were formed based on 2 periods (2000-2004 and 2005-2010). Information regarding bacteriological milk sample results from January 2000 through December 2010 was extracted from the Norwegian Dairy Herd Recording System (NDHRS) database. Only milk samples reported in the NDHRS to be collected because of suspicion or treatment of mastitis or as a follow-up of a mastitis case were used for environmental categorization. Each H5Y class was assigned to 1 of 2 environmental groups based on their prevalence of contagious pathogens (Staph. aureus, Strep. dysgalactiae, and Strep. agalactiae) in the bac- teriological milk samples analyzed. The H5Y classes were assigned to the environmental groups based on the percentage of milk samples found positive for contagious pathogens among all milk samples in the H5Y. The distribution of H5Y by frequency of milk samples containing contagious pathogens is shown in Figure 1. Herds were classified into groups in 2 different ways, based on their contagious pathogen prevalence: (1) low $50(\mathbf{L 5 0} ;<50 \%)$ and high $50(\mathbf{H} 5 \mathbf{5 0} ; \geq 50 \%)$, and $(2)$ low 75 (L75; $\leq 25 \%)$, medium 75 (M75; $>25 \%$ and $<75 \%)$, and high $75(\mathbf{H 7 5} ; \geq 75 \%)$. All H5Y with fewer than 5 milk samples and fewer than 5 first-lactation cows were excluded from the analyses. In addition, to ensure a certain amount of pathogen information, only H5Y with milk samples available for at least $10 \%$ of the first-lactation cows were included. In total, 6 data sets were constructed for the analyses, 1 for each environmental category (L50, H50, L75, M75, and H75) and 1 full data set (FD), including all data that was used in the comparisons. Only bulls with at least 5 daughters in each of the data sets were included for genomic analyses.

\section{Phenotypic Information}

The phenotype analyzed was the environment-specific DYD (an estimate of the average performance of each bull's progeny, corrected for all fixed and nongenetic random effects) for $\mathrm{CM}$ in the period from $15 \mathrm{~d}$ before to $30 \mathrm{~d}$ after first calving (CM30). The trait CM30 was chosen because it is one of the standard traits used in the routine genetic evaluation for Norwegian Red. For calculation of the DYD, CM records from firstlactation Norwegian Red cows calving from January 2000 through December 2009 were extracted from the NDHRS database. The cows were sired by progenytested and genotyped Norwegian Red AI bulls and were

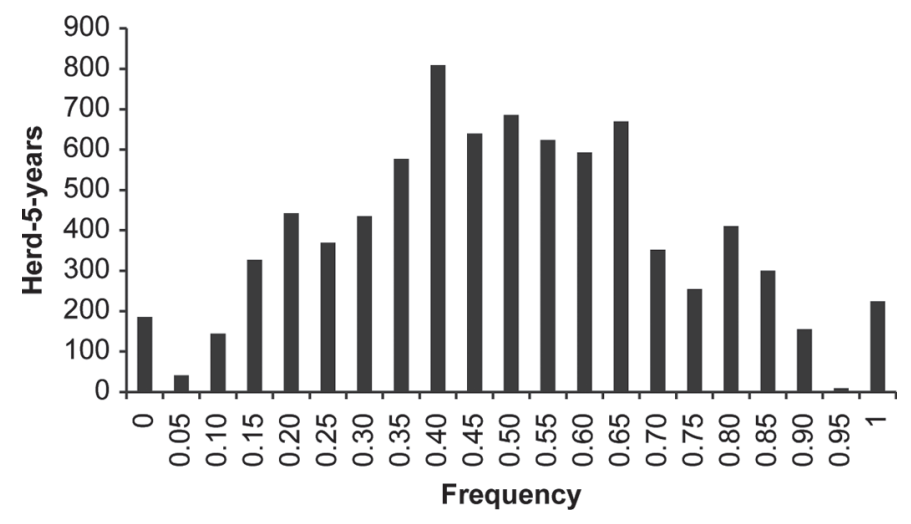

Figure 1. Frequency of contagious milk samples out of total milk samples within herd-5-years. 
20 to 36 mo old at first calving. The CM30 trait was scored as binary, where 1 indicated that the cow had one or more records of $\mathrm{CM}$ in the defined time period, and 0 otherwise. Data were analyzed using a univariate linear sire model. The model for an individual record of CM30 was

$$
\mathrm{CM} 0_{i j k l m}=a g e_{i}+m o \_y y_{j}+H 5 Y_{k}+\text { sire }_{l}+e_{i j k l m},[1]
$$

where CM30 ${ }_{i j k l m}$ is the observed CM30 of cow $m$; age $e_{i}$ is the fixed effect of age at first calving $(i=1-17$ singlemonth classes); mo_y $y_{j}$ is the fixed effect of month-year of calving $(j=1-108) ; H 5 Y_{k}$ is the random effect of herd-5-yr class $k$ (no. of levels varied with data set); sire $_{l}$ is the random effect of sire, and $e_{i j k l m}$ is the residual effect, $e_{i j k l m} \sim N\left(0, \sigma_{e}^{2}\right)$. A total of 3,304 bulls were included in the pedigree file, which consisted of the 1,126 bulls with daughters in the data set and their sires and the bulls' maternal grandsires, traced back as far as possible.

Variance components were estimated using the FD data set and the DMUAI procedure in DMU (Madsen and Jensen, 2007) which estimates the variance components using the average information REML algorithm (AI-REML; Meyer, 1997). The variance component estimates were used as true values for calculating DYD values in each of the data sets using the DMU5 procedure in DMU (Madsen and Jensen, 2007), which solves the mixed model equations numerically. The DYD for each bull was calculated by

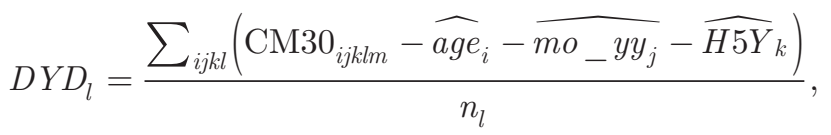

with notation as in equation [1] and where $n_{l}$ is the number of daughters with records for bull $l$.

\section{SNP Data}

An imputed $25 \mathrm{~K} / 54 \mathrm{~K}$ SNP data set for a total of 3,313 progeny-tested Norwegian Red sires was available (Solberg et al., 2011). This data set was created based on 2,165 bulls genotyped with the Affymetrix 25K chip (Affymetrix, San Diego, CA) and 1,575 bulls genotyped with the Illumina 54K chip (Illumina, San Diego, CA). Of these, 457 bulls were genotyped with both chips, and SNP were imputed both ways $(9,434$ common SNP). After editing and removal of SNP with minor allele frequency $<0.05,47,321$ SNP remained for the analyses. The marker genotypes were coded as $-1,0$, and 1 for homozygotes for the minor allele, heterozygotes, and homozygotes for the major allele, respectively.

\section{Model and Assessment of Predictive Ability}

Bayesian ridge regression (BRR) using the BLR package (de los Campos and Pérez, 2012) in R (R Development Core Team, 2009) was used to estimate SNP effects and to predict DYD. The BRR is equivalent to genomic BLUP (de los Campos et al., 2013). The linear regression model on markers can be written as

$$
D Y D_{l}=\mu+\sum_{g=1}^{p} x_{l g} \beta_{g}+e_{l}
$$

where $p$ is the number of markers, $\mu$ is the overall mean, $x_{l g}$ denotes the genotype at marker locus $g$ for sire $l$, the regression coefficient $\beta_{g}$ is the allele substitution effect of marker $g$, and $e_{l}$ is the model residual, $e_{l} \sim N\left(0, \frac{\sigma^{2}}{n_{l}}\right)$, where $n_{l}$ is the number of records used to compute $D Y D_{l}$. Note that the model has heterogeneous residual variances because we have different numbers of daughters per bull. The BRR shrinks marker effects toward zero, using a Gaussian prior with variance common to all markers; that is, $\beta_{g} \sim N\left(0, \sigma_{\beta}^{2}\right)$ (Pérez et al., 2010). The difference with BLUP of marker effects is that variance components are estimated from the training sample using a Bayesian approach implemented via Gibbs sampling. A scaled inverse Chi-squared distribution with degrees of freedom $d f_{\beta}$ and scale $S_{\beta}$ was assigned to $\sigma_{\beta}^{2}$. The values of the hyper-parameters $d f_{\beta}$ and $S_{\beta}$ were set to 4 and 1 , respectively. Predictive ability for CM30, within and between environmental groups, was evaluated using a 10 -fold cross validation. In this procedure, $90 \%$ of the sires, chosen randomly, were used for training and the remaining $10 \%$ were used for testing in the complementary data set (L50 and $\mathrm{H} 50$; and all the combinations among L75, M75, and H75). This was repeated 10 times to obtain an estimate of the cross-validation distribution of predictive ability. Because all sires were represented in all data sets and the folds were the same in all data sets (same sires), predictive ability could be obtained between the given DYD from one data set and a predicted DYD in another data set. To illustrate, $90 \%$ of the sires from L50 were used for training, with the testing done on the remaining $10 \%$ of the sires from L50 as well as testing on the same sires' DYD from H50. A 10-fold cross validation was also used for the FD. Inferences for each model-fitting process were based on 4,500 samples, obtained from total chain lengths of 45,000 samples plus 5,000 samples that were discarded as burn-in and with a thinning interval of 10 . Predictive ability was measured as Pearson correlation between the predicted DYD and the observed "true" DYD in the testing set. 
A bootstrap procedure (Efron and Tibshirani, 1993) was used to form confidence intervals $(\mathbf{C I})$ for predictive ability for each data set. A sire's observed DYD and its corresponding predicted DYD from the cross-validation procedure was assumed to be an independent draw from the same distribution, and the pair was sampled together at random in folds of 113 bulls. Predictive ability was then calculated for each fold. This was repeated 20,000 times within each environment, and a 95\% CI was formed from the sampled distribution.

\section{RESULTS AND DISCUSSION}

A description of the data sets is shown in Table 1. The CM30 frequency was similar in all data sets $(6.26$ to $7.10 \%$ ) with the lowest frequencies in L 75 . This data set had the highest average DYD (i.e., more mastitis) and highest maximum DYD value. The lowest DYD value (i.e., less mastitis) was almost the same in all data sets but was lowest, on average, in H75. The size of the daughter groups used to calculate DYD varied between the data sets. The minimum number of daughters per sire ranged from 5 to 33 , whereas the maximum ranged from 662 to 4,047 .

\section{Classification of Pathogens}

Categorization of pathogens into contagious and environmental groups is not definite and many pathogens have characteristics from both groups. The clear consensus is that Staph. aureus and Strep. agalactiae are contagious pathogens (e.g., Jain, 1979; Harmon, 1994); Strep. dysgalactiae, however, can occur as environmental in some herds and contagious in other herds (IDF, 1999). The results of this study may be affected by the chosen categorization of contagious mastitis pathogens. The classification of pathogens chosen in this study was in agreement with the common classification of contagious mastitis pathogens in Norway.

\section{Predictive Ability}

The mean (range) predictive ability for FD was 0.19 $(-0.02 ; 0.33)$. This was lower than previous genomeenabled predictions for CM30 in Norwegian Red (Heringstad et al., 2011; Solberg et al., 2011). Apart from the uncertainty in the cross-validation distribution, this was not surprising because the present study was based on CM30 data only from herds with pathogen information, resulting in a much smaller data set with fewer bulls. Predictive ability within and between environment for L50 and H50, and for L75, M75, and H75 are shown in Tables 2 and 3, respectively. Predictive ability was lower than when FD was used, with the mean correlation varying from 0.04 (L75) to 0.15 (H75).

The mean predictive ability for DYD within and between environments L50 and H50 ranged from 0.12 to 0.14 (Table 2). Training the model in one environment and testing it in the other (off-diagonal elements of Table 2) gave a similar predictive ability as that attained when training and testing the model in the same environment (diagonal elements in Table 2). Therefore, the results did not reveal any indication of $\mathrm{G} \times \mathrm{E}$ for mastitis in Norwegian Red, at least not for the current definition of environments. The uncertainty in the cross-validation distributions was large, suggesting that the data and layout may have lacked power for evaluating $\mathrm{G} \times \mathrm{E}$ in the context of this study.

Mean predictive ability for DYD varied more (0.04 to $0.15)$ when herds were classified in 3 groups according

Table 1. Number of sires, herd-5-yr (H5Y), and cows (total, minimum/sire, maximum/sire), mean frequency of CM30 (clinical mastitis from $15 \mathrm{~d}$ before to $30 \mathrm{~d}$ after first calving), and mean, minimum, and maximum values of daughter-yield-deviation (DYD) in the 6 data sets

\begin{tabular}{|c|c|c|c|c|c|c|}
\hline \multirow[b]{2}{*}{ Item } & \multicolumn{6}{|c|}{ Data set $^{1}$} \\
\hline & L50 & $\mathrm{H} 50$ & L75 & M75 & H75 & FD \\
\hline No. of sires & 1,126 & 1,126 & 1,126 & 1,126 & 1,126 & 1,126 \\
\hline No. of H5Y & 3,836 & 4,022 & 1,289 & 5,358 & 1,211 & 7.858 \\
\hline \% CM30 & 6.77 & 7.10 & 6.26 & 7.06 & 7.05 & 6.93 \\
\hline \multicolumn{7}{|l|}{ No. of cows } \\
\hline Total & 123,006 & 113,249 & 39,054 & 166,336 & 30,865 & 236,255 \\
\hline Minimum/sire & 11 & 15 & 6 & 18 & 5 & 33 \\
\hline Maximum/sire & 2,179 & 1,868 & 662 & 2,994 & 495 & 4,047 \\
\hline \multicolumn{7}{|l|}{ DYD } \\
\hline Mean & -0.003 & -0.006 & 0.0004 & -0.006 & -0.002 & -0.007 \\
\hline Minimum & -0.11 & -0.11 & -0.12 & -0.12 & -0.13 & -0.11 \\
\hline Maximum & 0.34 & 0.20 & 0.59 & 0.17 & 0.37 & 0.17 \\
\hline
\end{tabular}

${ }^{1}$ Herd-5-yr classes with the following prevalence of bacteriological milk samples positive for contagious pathogens: $<50 \%$ (L50), $\geq 50 \%$ (H50), $\leq 25 \%$ (L75), $>25 \%$ and $<75 \%$ (M75), and $\geq 75 \%$ (H75); FD is a data set with all data without considering environment. 
Table 2. Average [range] predictive abilities for daughter yield deviations of CM30 (clinical mastitis from $15 \mathrm{~d}$ before to $30 \mathrm{~d}$ after first calving) over all folds from a 10-fold cross-validation within (diagonal) and between (off diagonal) 2 environments with herds classified according to mastitis pathogen status ${ }^{1}$ (L50 and H50)

\begin{tabular}{|c|c|c|}
\hline \multirow[b]{2}{*}{ Testing } & \multicolumn{2}{|c|}{ Training } \\
\hline & L50 & H50 \\
\hline L50 & $0.12[-0.05 ; 0.32]$ & $0.14[-0.14 ; 0.30]$ \\
\hline H50 & $0.12[0.02 ; 0.21]$ & $0.13[-0.02 ; 0.22]$ \\
\hline
\end{tabular}

${ }^{1}$ Herd-5-yr classes with the following prevalence of bacteriological milk samples positive for contagious pathogens relative to total amount of bacteriological milk samples: $<50 \%$ (L50) and $\geq 50 \%$ (H50).

to mastitis pathogen status (L75, M75, H75; Table 3) than when they were classified in 2 groups. Both the best (H75) and the worst (L75) predictive abilities were found when training and testing the model in the same environment. Training the model using L75 resulted (on average) in lower predictive ability for testing in all 3 environments $(0.04-0.06)$ than when either M75 (0.08-0.13) or H75 (0.10-0.15) was used for model training. All 3 training sets had the lowest predictive ability when L75 was used as testing environment. These results indicate that selection based on performance in M75 and H75 would yield a higher response than selection based on performance in L75.

The lower correlations found when using L75 data, either for training or testing, suggest that CM30 data in herds with a low proportion of contagious mastitis pathogens may be less informative. If the assumption is that CM30 in H75 is caused by contagious pathogens and CM30 in L75 is caused by environmental pathogens, the fact that the latter group of pathogens is more diverse may result in heterogeneity of traits. The literature suggests that mastitis caused by different pathogens can be considered as genetically different traits (Sørensen et al., 2009; Haugaard et al., 2012), and $\mathrm{CM}$ caused by environmental pathogens tends to have higher heritability than CM caused by contagious pathogens.
Predictive ability varied greatly between folds and, in many situations, the range included zero (Tables 2 and 3). Box plots from the bootstrap distribution of the predictive ability from the 6 data sets showed large variation within each data set and small variation between the data sets (Figure 2), with the largest difference seen between FD and L75. Also, the 95\% CI formed from the bootstrapping procedure included zero; that is, no predictability, in 5 of the 6 data sets (Table 4). The exception was the full data set with a $95 \%$ CI of 0.02 to 0.35 . Interpretation of the results should therefore be made with great caution.

\section{SNP Effects}

Rank correlations for the SNP effects among the 6 data sets are shown in Table 5. Correlations ranged from 0.15 to 0.92 . The largest correlations were found between FD and L50, H50, and M75, whereas the lowest correlations were found among L75, M75, and H75. Of the 100 "best" SNP (less mastitis) in FD, 45, 31, 14, 54 , and 8 were also found among the 100 best SNP in L50, H50, L75, M75, and H75, respectively. Similarly, for the 100 "worst" SNP (more mastitis) in FD, 41, $37,9,48$, and 5 SNP were found among the worst 100 SNP in L50, H50, L75, M75, and H75, respectively. The low rank correlations between SNP effects in the various environments may indicate that SNP effects can be environment-specific. The highest correlations were found between the most "similar" environments, whereas the lowest correlations were found between the most "different" environments, which could be expected if pathogen-specific SNP effects exist. However, linkage disequilibrium between the SNP has not been taken into account here, and this may be another explanation for the low rank correlations.

\section{Environmental Groups}

Herds were classified into environmental groups based on prevalence of the contagious mastitis patho-

Table 3. Average [range] predictive abilities for CM30 (clinical mastitis from $15 \mathrm{~d}$ before to $30 \mathrm{~d}$ after first calving) over all folds from a 10-fold cross-validation within (diagonal) and between (off diagonal) environments with herd classified according to mastitis pathogen status ${ }^{1}$ (L75, M75 and H75)

\begin{tabular}{lccc}
\hline & \multicolumn{3}{c}{ Training } \\
\cline { 2 - 4 } Testing & L75 & M75 & H75 \\
\hline L75 & $0.04[-0.12 ; 0.18]$ & $0.08[-0.20 ; 0.27]$ & $0.10[-0.18 ; 0.23]$ \\
M75 & $0.06[-0.08 ; 0.18]$ & $0.13[0.04 ; 0.21]$ & $0.10[-0.11 ; 0.31]$ \\
H75 & $0.06[-0.03 ; 0.23]$ & $0.10[-0.02 ; 0.22]$ & $0.15[0.05 ; 0.42]$ \\
\hline
\end{tabular}

${ }^{1}$ Herd-5-yr classes with the following prevalence of bacteriological milk samples positive for contagious pathogens relative to total amount of bacteriological milk samples: $\leq 25 \%$ (L75), $>25 \%$ and $<75 \%$ (M75), and $\geq 75 \%$ (H75). 


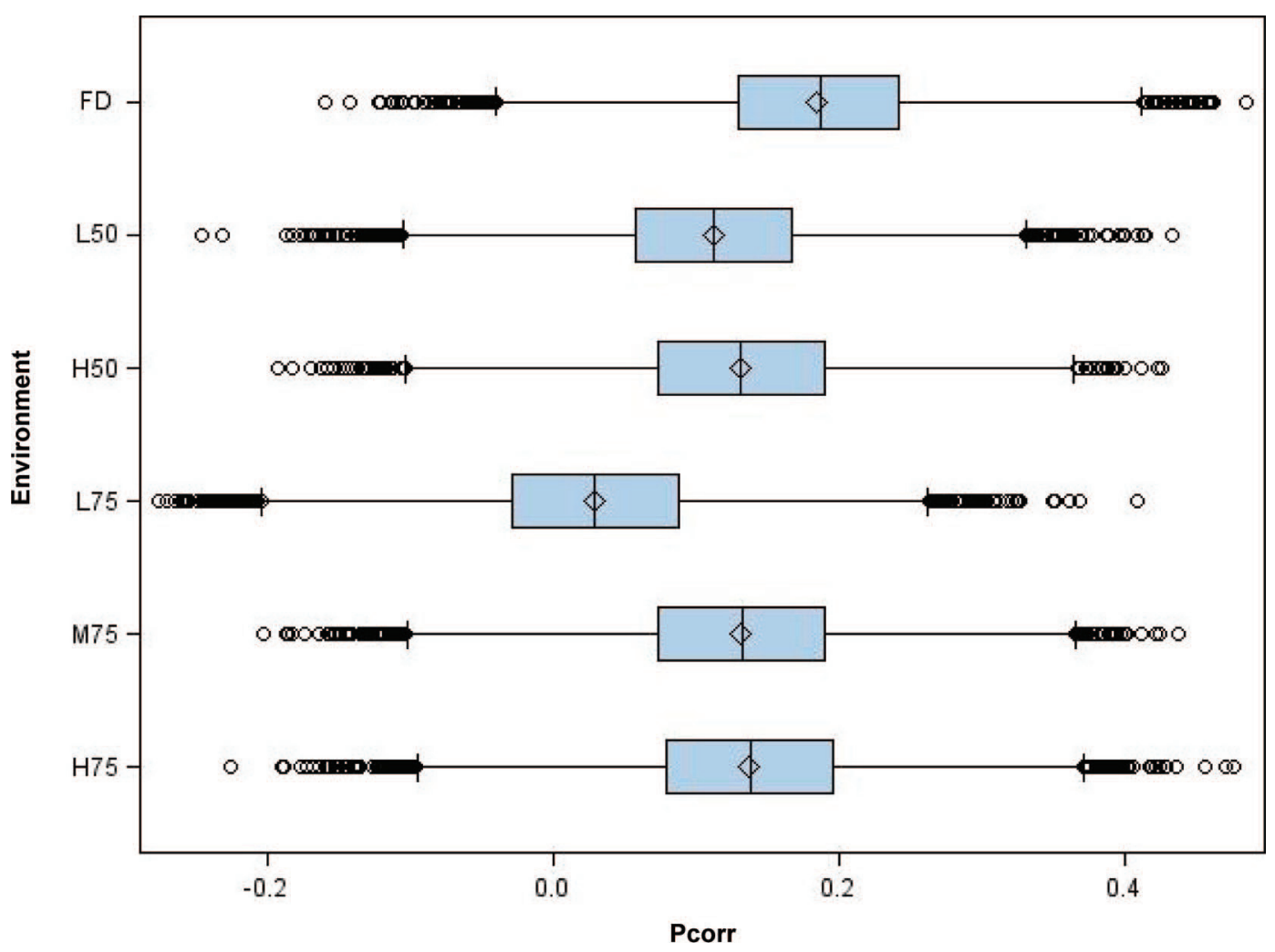

Figure 2. Box plots for the bootstrap distribution of the predictive ability (Pcorr) from the 6 data set. Each data set consisted of cows from herd-5-yr with the following prevalence of bacteriological milks samples positive for contagious pathogens relative to total amount of bacteriological milk samples: $<50 \%$ (L50), $\geq 50 \%$ (H50), $\leq 25 \%$ (L75), $>25 \%$ and $<75 \%$ (M75), and $\geq 75 \%$ (H75); FD is a data set with all data without regard to environment. Color version available in the online PDF.

gens Staph. aureus, Strep. dysgalactiae, and Strep. agalactiae in milk samples. This group of pathogens was chosen because it is the most prevalent in mastitis milk samples analyzed at Norwegian mastitis laboratories. Two sets of thresholds were used to investigate the differences in environmental definition. The $50 \%$ threshold was chosen as a basis, splitting both the data set and the number of herds approximately in half. However, it can be questioned how different the H5Y

Table 4. Mean, SD, and $95 \%$ confidence interval (CI) of the bootstrap distribution of the predictive ability in the 6 data sets

\begin{tabular}{lccc}
\hline Data set $^{1}$ & Mean & SD & $95 \%$ CI \\
\hline FD & 0.18 & 0.08 & {$[0.02 ; 0.35]$} \\
L50 & 0.11 & 0.08 & {$[-0.05 ; 0.27]$} \\
H50 & 0.13 & 0.09 & {$[-0.04 ; 0.29]$} \\
L75 & 0.03 & 0.09 & {$[-0.14 ; 0.20]$} \\
M75 & 0.13 & 0.09 & {$[-0.04 ; 0.29]$} \\
H75 & 0.14 & 0.09 & {$[-0.03 ; 0.30]$} \\
\hline
\end{tabular}

${ }^{1}$ Herd-5-yr classes with the following prevalence of bacteriological milk samples positive for contagious pathogens relative to total amount of bacteriological milk samples: $<50 \%$ (L50), $\geq 50 \%$ (H50), $\leq 25 \%$ (L75), $>25 \%$ and $<75 \%$ (M75), and $\geq 75 \%$ (H75); FD is a data set with all data without regard to environment. in L50 and H50 truly are. The distribution of H5Y by proportion of milk samples with contagious mastitis pathogens was largely symmetric (Figure 1). A large proportion of $\mathrm{H} 5 \mathrm{Y}$ was concentrated around $50 \%$, so many H5Y, in spite of similar contagious pathogen status, were classified into different environmental groups. The similar predictive ability within and between H50 and L50 (Table 2) may reflect that this environmental definition was not able to capture relevant differences with respect to either health or production environments. This issue was taken into consideration in the $75 \%$ data set, where $\mathrm{H} 5 \mathrm{Y}$ were classified into 3 groups and the high and low groups were of main interest. The difference in predictive ability between L75 and H75 (Table 3) suggests that some environmental difference was captured in this scheme. The threshold was set at $75 \%$, because empirical evaluation of the data indicated that a higher threshold would produce a data set that was too small for meaningful analysis.

\section{Daughter Yield Deviation}

The phenotypic trait to be predicted was DYD for CM30. This trait was chosen because CM30 is one of 
Table 5. Spearman rank correlations for SNP effects in the 6 data sets ${ }^{1}$

\begin{tabular}{llllll}
\hline & FD & L50 & H50 & L75 & M75 \\
\hline L50 & 0.84 & & & & \\
H50 & 0.82 & 0.42 & & & \\
L75 & 0.54 & 0.66 & 0.25 & & \\
M75 & 0.92 & 0.76 & 0.77 & 0.30 & 0.26 \\
H75 & 0.49 & 0.23 & 0.61 & 0.15 & 0.26 \\
\hline
\end{tabular}

${ }^{1}$ Herd-5-yr classes with the following prevalence of bacteriological milk samples positive for contagious pathogens relative to total amount of bacteriological milk samples: $<50 \%$ (L50), $\geq 50 \%$ (H50), $\leq 25 \%$ (L75), $>25 \%$ and $<75 \%$ (M75), and $\geq 75 \%$ (H75); FD is a data set with all data without regard to environment.

the traits used for routine genetic evaluation of mastitis resistance in Norway, and DYD is the average of the daughters' performance corrected for fixed and random nongenetic effects. As only information from the daughters is used, each DYD is computed independently from each other. Alternatively, EBV are based on information from all known relatives of the bulls, and the EBV of a given bull is dependent on information from other bulls (e.g., brothers, sons, and sires).

The mean number of daughters per sire was low (Table 1) relative to the daughter groups used for progeny testing in Norwegian Red, and thus the precision of DYD was poorer for the sub data sets. This level of precision (Tables 2 and 3) is therefore not representative of what could be obtained when using data from the whole population. To receive their first official proofs, Norwegian Red bulls are required to have at least 150 daughters with mastitis data, and the average daughter group for the first-crop evaluation of bulls is approximately 250 . In the FD data set used here, the average number of daughters per bull was 209, but $65 \%$ of the bulls had fewer than 100 daughters in the data set. The correlation between predicted and true DYD for the 137 bulls with 150 daughters or more in FD was 0.46 for FD and ranged from 0.20 to 0.35 for the environmental data sets.

\section{Random Cross Validation}

Random cross validation has a tendency to produce higher correlations than, for example, grouping the bulls according to age, as in applied use of genomic selection. In the cross validation, bulls were randomly assigned to 1 of 10 folds. This does not take population structure into account. In applied genomic selection, older proven bulls will be used as the reference population and the younger animals will be in the testing set. With random cross validation, a son can be used as reference for his father, something that will not happen in applied genomic selection. Accounting for population structure would probably give lower predictions than presented here, whereas using more data would give better predictions.

\section{CONCLUSIONS}

The aim of this study was to investigate whether $\mathrm{G} \times \mathrm{E}$ effects exist for CM in Norwegian Red. No indication of such interaction was provided, at least not for the environment definitions used here. However, rank correlations between the SNP effects were low for some data sets, indicating that various SNP are important in different environments.

\section{ACKNOWLEDGMENTS}

The authors thank the Norwegian Dairy Herd Recording System, the Norwegian Cattle Health Services (Ås, Norway) and Geno Breeding and AI Organization (Hamar, Norway) for access to phenotypic and genotypic data, and the Fulbright Foundation Norway (Oslo, Norway) for financial support.

\section{REFERENCES}

Burgueno, J., G. de los Campos, K. Weigel, and J. Crossa. 2012. Genomic prediction of breeding values when modeling genotype $\mathrm{x}$ environment interaction using pedigree and dense molecular markers. Crop Sci. 52:707-719.

Calvinho, L. F., R. A. Almeida, and S. P. Oliver. 1998. Potential virulence factors of Streptococcus dysgalactiae associated with bovine mastitis. Vet. Microbiol. 61:93-110.

de los Campos, G., J. M. Hickey, R. Pong-Wong, H. D. Daetwyler, and M. P. L. Calus. 2013. Whole genome regression and prediction methods applied to plant and animal breeding. Genetics 193:327345. http://dx.doi.org/10.1534/genetics.112.143313.

de los Campos, G., and P. Pérez. 2012. BLR: Bayesian linear regression. $\mathrm{R}$ package, version 1.3. http://www.r-project.org/.

Efron, B., and R. J. Tibshirani. 1993. An Introduction to the Bootstrap. Chapman and Hall, New York, NY.

Falconer, D. S. 1952. The problem of environment and selection. Am. Nat. 86:293-298.

Falconer, D. S. 1960. Selection of mice for growth on high and low planes of nutrition. Genet. Res. 1:91-113.

Falconer, D. S., and M. Latyszewski. 1952. The environment in relation to selection for size in mice. J. Genet. 51:67-80.

Harmon, R. J. 1994. Physiology of mastitis and factors affecting somatic cell counts. J. Dairy Sci. 77:2103-2112.

Haugaard, K., B. Heringstad, and A. C. Whist. 2012. Genetic analyses of pathogen-specific clinical mastitis in Norwegian Red cows. J. Dairy Sci. 95:1545-1551.

Heringstad, B., G. Su, T. R. Solberg, B. Guldbrandtsen, M. Svendsen, and M. S. Lund. 2011. Genomic predictions based on a joint reference population for Scandinavian red breeds. Book of Abstracts 
No. 17:29 in 62nd Annu. Mtg. EAAP, Stavanger, Norway. EAAP, Rome, Italy.

IDF (International Dairy Federation). 1999. Suggested interpretation of mastitis terminology. IDF Bull. 321. IDF, Brussels, Belgium.

Jain, N. C. 1979. Common mammary pathogens and factors in infection and mastitis. J. Dairy Sci. 62:128-134.

Lillehammer, M., M. E. Goddard, H. Nilsen, E. Sehested, H. G. Olsen, S. Lien, and T. H. E. Meuwissen. 2008. Quantitative trait locusby-environment interaction for milk yield traits in Bos taurus autosome 6. Genetics 179:1539-1546.

Long, N., D. Gianola, G. J. M. Rosa, K. A. Weigel, and S. Avendaño. 2008. Marker-assisted assessment of genotype by environment interaction: A case study of single nucleotide polymorphism-mortality association in broilers in two hygiene environments. J. Anim. Sci. 86:3358-3366.

Madsen, P., and J. Jensen. 2007. A user's guide to DMU: A package for analyzing multivariate mixed models. University of Aarhus, Research Center, Foulum, Tjele, Denmark.

Meyer, K. 1997. An 'average information' restricted maximum likelihood algorithm for estimating reduced rank genetic covariance matrices or covariance functions for animal models with equal design matrices. Genet. Sel. Evol. 29:97-116.

Neuman, R. J., J. Wasson, G. Atzmon, J. Wainstein, Y. Yerushalmi, J. Cohen, N. Barzilai, I. Blech, B. Glaser, and M. A. Permutt. 2002. Gene-gene interactions lead to higher risk for development of type 2 diabetes in an Ashkenazi Jewish population. PLoS ONE 5:e9903.

Pérez, P., G. de los Campos, J. Crossa, and D. Gianola. 2010. Genomic-enabled prediction based on molecular markers and pedigree using the Bayesian Linear Regression package in R. Plant Genome $3: 106-116$.

R Development Core Team. 2009. R: A language and environment for statistical computing. R Foundation for Statistical Computing, Vienna, Austria. http://www.r-project.org/.

Solberg, T. R., B. Heringstad, M. Svendsen, H. Grove, and T. H. E. Meuwissen. 2011. Genomic predictions for production- and functional traits in Norwegian Red from BLUP analyses of imputed 54K and 777K SNP data. Interbull Bull. 44:240-243.

Sørensen, L. P., B. Guldbrandtsen, J. R. Thomasen, and M. S. Lund. 2008. Pathogen-specific effects of quantitative trait loci affecting clinical mastitis and somatic cell count in Danish Holstein cattle. J. Dairy Sci. 91:2493-2500.

Sørensen, L. P., P. Madsen, T. Mark, and M. S. Lund. 2009. Genetic parameters for pathogen-specific mastitis resistance in Danish Holstein cattle. Animal 3:647-656.

Wang, X., B. Zuckerman, C. Pearson, G. Kaufman, C. Chen, G. Want, T. Niu, P. H. Wise, H. Bauchner, and X. Xu. 2002. Maternal cigarette smoking, metabolic gene polymorphism, and infant birth weight. JAMA 287:195-202.

Wei, X., P. A. Jackson, S. Hermann, A. Kilian, K. Heller-Uszynska, and E. Deomano. 2010. Simultaneously accounting for population structure, genotype by environment interactions, and spatial variation in marker-trait associations in sugarcane. Genome 53:973981. 